\title{
Optimization of Cryo-EM Data Collection Using Advanced Direct Detectors
}

\author{
Michael Spilman \\ Direct Electron, LP, San Diego, California, United States
}

The development of direct detection cameras for transmission electron microscopy has enabled recording of images with unprecedented quality. These large-format ( $>4$ million pixels) cameras can deliver a continuous stream of images with near-optimal detective quantum efficiency (DQE). Notably, this development led to the "resolution revolution" [1] in biological electron cryo-microscopy (cryo-EM), culminating in the 2017 Nobel Prize in Physics for the development of this technique. The latest generation of direct detectors have numerous improvements in speed, sensitivity, and array size that offer increased flexibility and throughput as microscope time has become more competitive with large facilities requiring preliminary data. Typically slow techniques such as cryo-electron tomography (cryo-ET) [2] and microcrystalline electron diffraction (microED) [3] have seen significant speed increases by coupling a continuously tilting stage with synchronized readout from the detector.

In this tutorial, I will cover how to utilize the latest generation of direct detection cameras to optimize data collection for several cryoEM techniques including single particle, tomography, and microED. While single particle data collection schemes typically follow a fairly standard set of parameters based on camera speed and electron dose rate, I will cover the benefits of large pixel counting using hardware binning to maximize data quality and when linear mode makes sense in terms of resolution targets and throughput using $8 \mathrm{k} \mathrm{x} \mathrm{8k}$ arrays. I will also discuss two methods that benefit from the latest generation hardware: continuous-tilt tomography and microED. Continuous-tilt methods benefit from global shutter readout synchronous readout of all pixels in a frame. Automation of these methods significantly increases the throughput compared to traditional discreet-angle tilt methods. I will also highlight some of the challenges to using direct detectors with these techniques and how they can be addressed.

\section{References}

[1] W Kühlbrandt, Science 343 (2014), p. 1443.

[2] G Chreifi, S Chen, LA Metskas, M Kaplan, GJ Jensen. J. Struct. Biol., 205 (2019), pp. 163-169.

[3] Shi D., Nannenga B.L., Iadanza M.G., Gonen T. (2013). Elife, 2: e01345. 